\title{
Microscale and Nanoscale Electrophotonic Diagnostic Devices
}

\author{
Kaiyu Fu, ${ }^{1}$ Wei Xu, ${ }^{2}$ Jiayun Hu, ${ }^{1}$ Arielle Lopez, ${ }^{1}$ and Paul W. Bohn ${ }^{1,3}$ \\ ${ }^{1}$ Department of Chemistry and Biochemistry, University of Notre Dame, Notre Dame, Indiana 46556 \\ ${ }^{2}$ Advanced Light Source, Lawrence Berkeley National Laboratory, Berkeley, California 94720 \\ ${ }^{3}$ Department of Chemical and Biomolecular Engineering, University of Notre Dame, Notre Dame, \\ Indiana 46556 \\ Correspondence: pbohn@nd.edu
}

\begin{abstract}
Detecting and identifying infectious agents and potential pathogens in complex environments and characterizing their mode of action is a critical need. Traditional diagnostics have targeted a single characteristic (e.g., spectral response, surface receptor, mass, intrinsic conductivity, etc.). However, advances in detection technologies have identified emerging approaches in which multiple modes of action are combined to obtain enhanced performance characteristics. Particularly appealing in this regard, electrophotonic devices capable of coupling light to electron translocation have experienced rapid recent growth and offer significant advantages for diagnostics. In this review, we explore three specific promising approaches that combine electronics and photonics: (1) assays based on closed bipolar electrochemistry coupling electron transfer to color or fluorescence, (2) sensors based on localized surface plasmon resonances, and (3) emerging nanophotonics approaches, such as those based on zero-mode waveguides and metamaterials.
\end{abstract}

$T^{\text {him }}$ he first line of defense against the scourge of infectious agents centers on detection of pathogens at the point of care ( $\mathrm{PoC})$. Consequently, detecting and identifying infectious agents have attracted an enormous amount of attention, and molecular diagnostics, particularly those that exploit recent advances in nucleic acid detection technology (e.g., polymerase chain reaction [PCR] [Elnifro et al. 2000], highthroughput sequencing [Shendure and Ji 2008], etc.), have come to the fore. At the same time, there is a general realization that nucleic acid amplification brings with it the unwanted bag- gage of increased diagnostic complexity, prolonged analysis times, and even problems with clinical sensitivity and selectivity (Jung et al. 2010). Furthermore, genetic analyses are insufficient to address general pathogen challenges, because pathogen phenotype is not uniquely determined by the genotype alone. Therefore, a fully competent analysis should address the complete spectrum of pathogen characteristics, including the abilities to (1) locate and adhere to host tissues; (2) harm the host, for example, by exotoxin secretion; and (3) evade the immune system of the host. These are especially

Editors: Valentin A. Pavlov and Kevin J. Tracey

Additional Perspectives on Bioelectronic Medicine available at www.perspectivesinmedicine.org

Copyright (C) 2019 Cold Spring Harbor Laboratory Press; all rights reserved; doi: 10.1101/cshperspect.a034249

Cite this article as Cold Spring Harb Perspect Med 2019;9:a034249 
K. Fu et al.

important in light of new emerging pathogens. Thus, developing methods to rapidly and accurately identify potential pathogens in complex environments and to characterize their mode of action is critical, and the technological figures-of-merit that must be achieved are daunting.

Against this backdrop, a number of new technologies have been thrown into the breach, including both whole-cell detection and molecular recognition. For example, surface capture of bacteria is a mainstay in a number of assay formats in which different capture agents (lectins, antibodies, recognition peptides, aptamers, etc.) have been used to pull down bacteria to a surface. Once captured, the bacterium can be detected, for example, by fluorescence (Delehanty and Ligler 2002), surface plasmon resonance (Fratamico et al. 1998), optical waveguide resonance (RoweTaitt et al. 2000), amperometry (Gau et al. 2001), surface-enhanced Raman scattering (Grow et al. 2003), optical resonators (Watts et al. 1994), mass spectrometry (Bundy and Fenselau 1999), and electrochemical impedance (Varshney et al. 2007). Whereas these approaches are useful, they typically exploit a single mode of action. At the same time, a new set of detection technologies is emerging in which multiple modes of action are combined to yield detection schemes with enhanced performance characteristics. In particular, microscale and nanoscale electrophotonic devices, structures capable of coupling light to electron transfer and/or translocation, have experienced rapid growth over the last decade and offer significant advantages for diagnostic platforms. In this review, we will highlight three new approaches to combining electronics and photonics, which have substantial diagnostic promise: (1) closed bipolar electrochemistry (Liu et al. 2016b) coupling electron transfer to color, fluorescence, etc.; (2) localized surface plasmon resonance (LSPR) sensors (Shinohara et al. 2015); and (3) emerging nanophotonics approaches, such as those based on nonclassical optics, such as zero-mode waveguides (ZMWs) (Eid et al. 2009) and metamaterials (Hendry et al. 2010).

\section{BACKGROUND}

\section{Bipolar Electrochemistry \\ General Principles}

Bipolar electrochemistry, which couples redox reactions at the opposite poles of a nonpowered (i.e., unconnected) metal in contact with an electrified fluid, has opened new avenues of electrochemical sensing (Fig. 1A) (Fosdick et al. 2013). Two driving electrodes at opposite ends of the fluid medium produce a potential gradient in electrolyte solution, which maps onto the isopotential of the bipolar electrode (BPE), creating interfacial potential differences between the opposite poles of the BPE and the electrolyte solution in contact with them (Mavre et al.2010). This potential difference enables the coupling of anodic and cathodic reactions at the two ends (poles) of the BPE. Because BPEs can be fabricated in a variety of sizes, shapes, and topological forms, numerous applications have been described, including material preparation and fabrication (Ulrich et al. 2008), molecular sensing (Fosdick and Crooks 2012), microswimmers (Wang et al. 2006), and BPE focusing (Perdue et al. 2009).

Open BPEs (Fig. 1A) and closed BPEs (CBEs) (Fig. 1B) have both been explored (Fosdick et al. 2013). Open BPEs typically employ a single channel, and the anodic and cathodic poles are in the same solution environment, with coupled redox reactions occurring at the opposite poles (Mavre et al. 2010). In this geometry, ionic current constitutes a large fraction of the total current between driving electrodes. Because there is no external connection, BPE arrays, consisting of a large number of individual BPE electrodes, can be controlled with one set of driving electrodes (Chow et al. 2009). Therefore, open BPEs are good candidates for wireless electrochemical sensing in fluidic environments. In contrast, CBEs are analogous to series-coupled electrochemical cells (Guerrette et al. 2012). The anodic and cathode poles in $\mathrm{CBE}$ systems are in contact with physically separate (and possibly chemically distinct) solutions. Because the two compartments are physically separated, the BPE provides the only path for electron transfer between the two coupled 
A

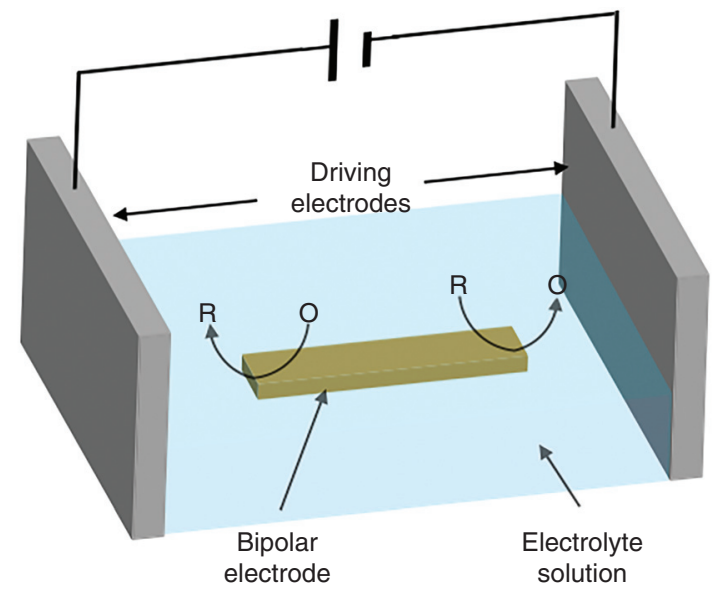

B

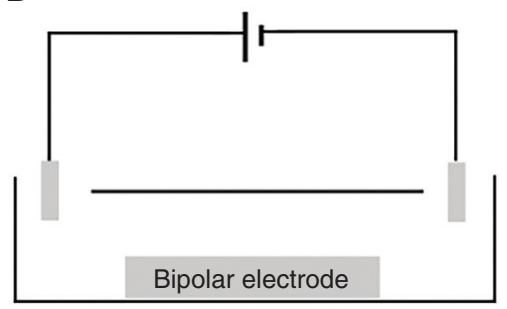

C

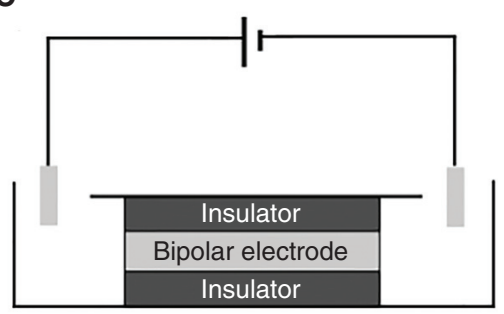

Figure 1. Bipolar electrochemistry is accomplished when a metal at open circuit is placed in contact with an electrified fluid, such that an interfacial potential is developed at both ends-positive at one end and negative at the other. (A) Schematic illustration of the working principle of bipolar electrochemistry. An unconnected metal is placed in an electrified fluid and coupled cathodic (left) and anodic (right) reactions are driven by the potential offset between the electrode pole and the contacting solution. Experimental configurations for open $(B)$ and closed $(C)$ bipolar electrodes (BPEs). Note that in $C$, the two solutions contacting the BPE are completely isolated and can, thus, be of different compositions.

half reactions (Guerrette et al. 2012). Therefore, CBEs may employ chemically distinct analytes and reporters, thus physically segregating the detection from readout steps, greatly minimizing background interference. Also, the reporter system can be regenerated and reused many times. Thus, CBEs can implement remote detection (Xu et al. 2016a), reducing cross talk between analyte and reporter solutions and eliminating the need for a fluidic path containing the BPE (Xu et al. 2016b).

\section{Optical Readout Strategies}

Although early bipolar electrochemistry research largely focused on coupling redox reactions and measuring the resulting Faradaic current (Loget et al. 2013), recent applications have demonstrated that converting electric to optical signals can be a powerful approach to improve sensitivity, simplify instrumentation design, and suppress signal cross talk (Zhang et al. 2017). Here, we will highlight recent developments using optical readout strategies, including those based on electrochromic, electrofluorigenic, and electrochemical plasmon effects, all of which allow electron transfer at one pole of a CBE to be read out by an optical reporter system at the other pole.

Electrochromic readout is achieved when an electron transfer reaction in the reporter cell results in a colorimetric change in the CBE (Liu and Crooks 2012). For example, we developed an electrochromic-sensing platform based on coupling redox reactions in an analytical cell 
K. Fu et al.

with a redox-sensitive colorimetric indicator, methyl viologen (MV), in the reporter cell (Xu et al. 2016a). The whole detection system was designed to work with battery power and cellphone color readout as a proof-of-concept of device simplicity and portability. Electrofluorigenic readout can be realized by exploiting redox-sensitive fluorescence (e.g., in resorufin), to achieve ultrasensitive detection. In this approach, redox reactions in the analytical cell were coupled to the electrochemical transformation of weakly emissive resazurin to strongly emissive resorufin in the reporter cell. The effect is versatile, as was demonstrated in two different geometries: nanopore recessed disk-multiscale BPEs (Ma et al. 2015) and parallel interdigitated electrode BPEs. Besides the high sensitivity, both detection systems are capable of fluorescence imaging, which can be used to explore heterogeneous electrochemical phenomena. Looking to the future, we are developing a CBE approach that uses LSPR readout to combine the simplicity of electrochromic assays and the sensitivity of electrofluorigenic assays. In this strategy, simple galvanic electrodeposition is used to shift the LSPR wavelength to monitor the reaction in the analytical cell.

\section{Localized Surface Plasmon Resonances \\ General Principles}

LSPRs constitute an optical effect observed particularly in noble metal (e.g., $\mathrm{Au}, \mathrm{Ag}, \mathrm{Cu}$ ) nanoparticles, which possess a dielectric constant with a negative real and a small positive imaginary component (Willets and Van Duyne 2007). When the frequency of incident radiation matches the resonant oscillation frequency of free electrons in the nanoparticles, electrons oscillate coherently (Fig. 2A), resulting in a surface plasmon resonance. Because the nanoparticles size is typically $\leq \lambda / 10$, the induced resonance is highly localized around the metal nanoparticles (i.e., an LSPR).

\section{Construction and Characterization}

Metallic nanoparticles can be chemically synthesized in solution as well as patterned onto substrates using lithography (Willets and Van Duyne 2007). Combining the advantages of these two methods, nanosphere lithography (NSL) (Hulteen and Van Duyne 1995) allows large-scale fabrication of periodic arrays of metallic nanoparticles. Modified NSL methods have been used to create different patterns on substrates such as modulated nanopore arrays (Fu et al. 2016) and nanopillar arrays (Hall et al. 2013). NSL fabrication makes ideal substrates for LSPR spectroscopy (Haes and Van Duyne 2004). The extinction of metal nanoparticle arrays is monitored using a UVvisible spectrometer in either transmission or reflection. The LSPR can be tuned based on the material, size, and shape of the nanoparticles, as well as the surrounding environment, including both solvent and adsorbates. This sensitivity to the environment has been exploited for biomolecular-sensing applications (Hall et al. 2011a), with the key being the manipulation of the local dielectric environment and the resulting shift in the LSPR wavelength, $\Delta \lambda$ (Fig. 2B).

\section{Selectivity}

LSPR sensing is inherently nonspecific; anything adsorbed onto the sensor surface can shift the LSPR wavelength. Thus, to create a selective LSPR-sensing platform, recognition agents with high affinities toward their specific targets are incorporated in the sensor design. A broad range of recognition agents include biotin and (strept/neutr) avidin (Haes and Van Duyne 2002), antibody-antigen pairs (Hall et al. 2011b), lectin and carbohydrates (Cai et al. 2017), enzyme-substrate pairs (Sekretaryova et al. 2014), siderophores (Doorneweerd et al. 2010), bacteriophages (Tripathi et al. 2012), as well as oligonucleotide-based recognition systems, such as aptamers (Urmann et al. 2016), antimicrobial peptides (Mannoor et al. 2010), and other DNA sequences. These recognition agents are used to target small molecules $(\mathrm{Mu}-$ guruma et al. 2011), proteins (Bertok et al. 2013), bacterial lysates (Taylor et al. 2006), bacterial spores (Pestov et al. 2008), intact bacterial cells (Hu et al. 2018), viral proteins (Nidzworski 
A

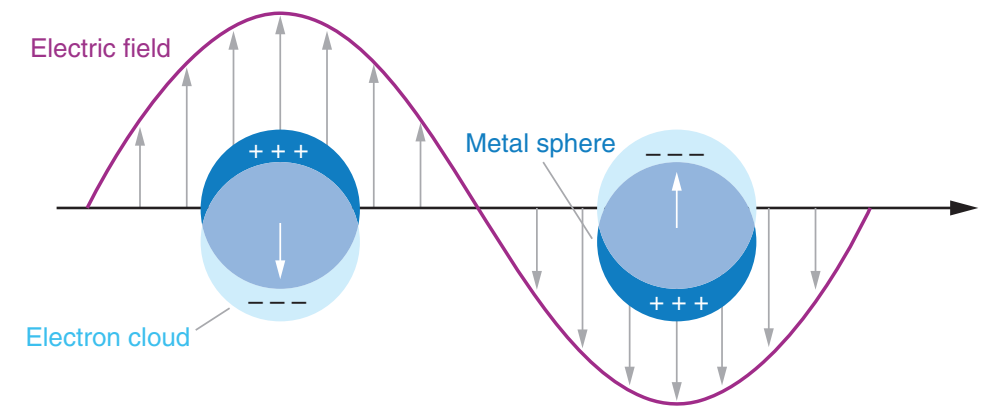

B

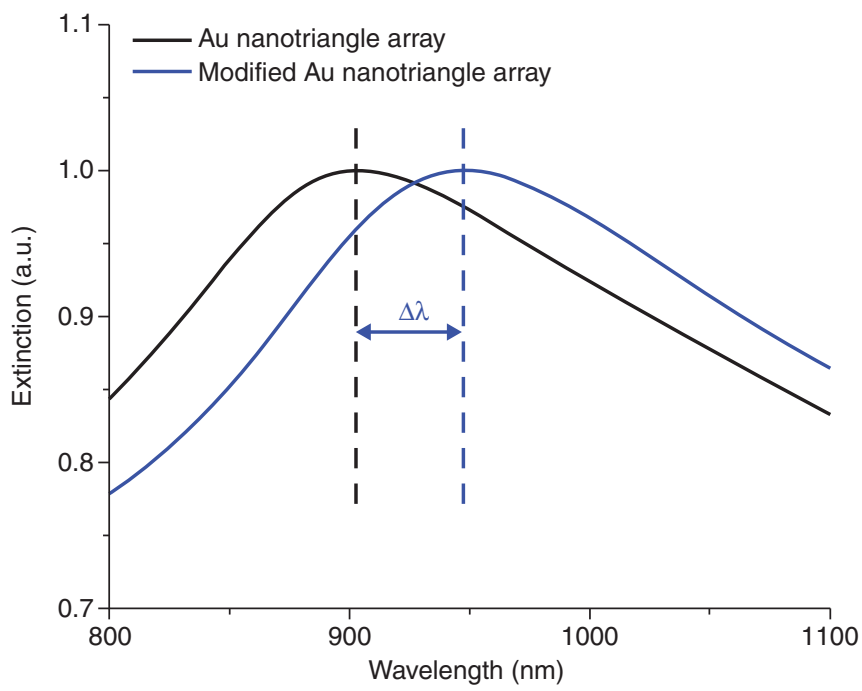

Figure 2. Localized surface plasmon resonance (LSPR)-based sensing takes advantage of the sensitivity of free electron oscillations in a metallic nanoparticle to the dielectric environment just outside the particle. (A) Schematic diagram illustrating the origin of LSPRs. $(B)$ Wavelength shift resulting from a change in the dielectric environment around the LSPR particle used in biomolecular sensing. (Panel $A$ modified from Willets and Van Duyne 2007; adapted, with permission, from Annual Reviews (C) 2007.)

et al. 2017), viral DNAs (Dong et al. 2015), and intact viruses (Chang et al. 2018).

\section{Nanophotonics}

At the extreme sensitivity limit, the direct electrochemical observation of single-electron transfer events in biological systems is a severe challenge. The problem arises because the generated currents are at or below the noise floor of the measurement. Thus, the principal approaches to measuring single molecules electrochemically have relied on amplifying the signal, for example by redox cycling (Zevenbergen et al.
2007). Alternatively, converting redox processes to photon emission events (i.e., luminescence) can circumvent the noise floor problem, a fact that was exploited by Xie, who studied single redox enzymes, using the redox-sensitive florescence of flavin adenine dinucleotide (FAD) to monitor enzyme turnover in single molecules of cholesterol oxidase (Lu and Xie 1999).

Although the electrochemical behavior of single molecules has been characterized, observing single-electron transfer events is a much more challenging objective (Byers et al. 2015). Hoeben et al. observed single-enzyme electrochemistry in nanolithographic structures pro- 
K. Fu et al.

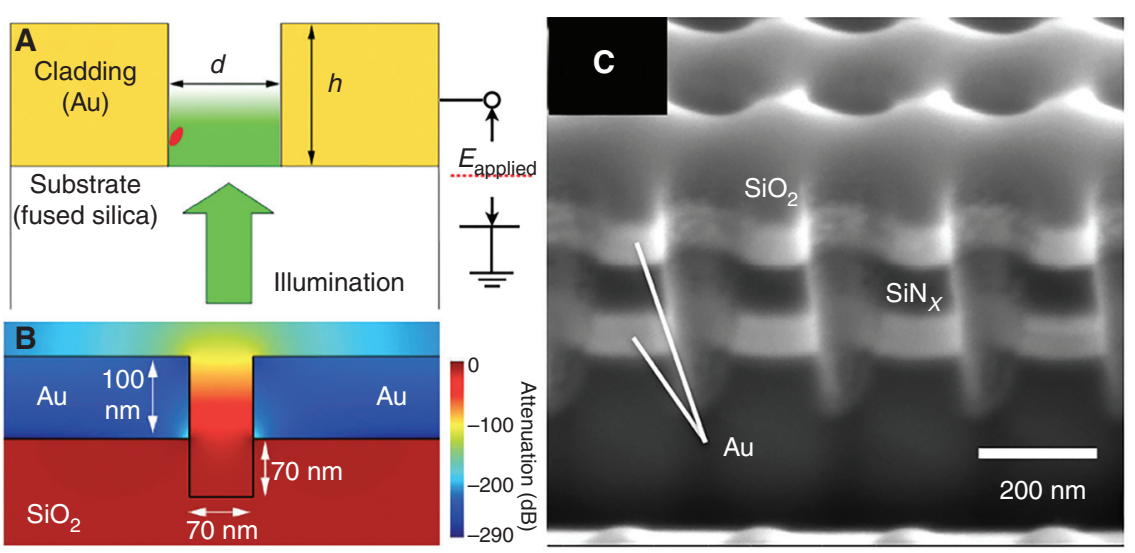

Figure 3. The electrochemical zero-mode waveguide (E-ZMW) is a nanoscale architecture that combines the optical field confinement of a zero-mode waveguide, with the ability to control the electrochemical potential at the optical cladding layer, which is used as a working electrode. (A) Schematic cross-sectional diagram of a single E-ZMW nanopore illustrating the multifunctional Au film used as a working electrode and optical cladding layer. Red ellipse represents a single copy of an enzyme. (B) Cross-sectional schematic of a single ZMW nanopore with optical field heat map. $(C)$ Cross-section structural equation modeling (SEM) image of three adjacent E-ZMW nanopores showing dual recessed $\mathrm{Au}$ ring electrodes separated by $\sim 100 \mathrm{~nm}$ of $\mathrm{SiN}_{x}$. The bottom $\mathrm{Au}$ ring constitutes the optical cladding layer of the E-ZMW.

ducing fA-scale currents commensurate with the electron transfer activity of $\sim 10$ molecules (Hoeben et al. 2008). They pioneered the use of a nanometer-spaced anode and cathode on opposite faces of a nanofluidic channel, allowing them to observe correlations between successive single-molecule electron transfer events (Zevenbergen et al. 2009).

Another kind of nanophotonic platformthe electrochemical ZMW (E-ZMW)-consists of a small $(d<100 \mathrm{~nm}$, typ. $)$ cylindrical perforation in a thin metal film, capable of trapping optical radiation (see Fig. 3; Levene et al. 2003). The trapped radiation can interact with molecules contained in the zeptoliter-scale $(1 \mathrm{zL}=$ $10^{-21} \mathrm{~L}$ ), volume bounded by the radiation field within the nanopore. The small volumes enclosed by the active region of the E-ZMW, their close proximity to the working electrode, and the small distances over which diffusive mass transport occurs, combine to give the E-ZMW some powerful properties, including the ability to observe single-electron transfer events using simultaneous optical and electrochemical measurements. Although ZMWs may be constructed as single nanopores, more commonly they are fabricated as a parallel array of nanopores, which is advantageous, because (1) the average occupancy of individual pores may be controlled by concentration, (2) the electrodes in each individual pore are connected to those in all the other pores, so they can all be controlled at the same potential, $E_{\text {appl }}$, and (3) the electrochemical behavior is integrated over the entire array. The central characteristic of these architectures is that single-molecule spectroscopic and electrochemical data can be acquired simultaneously. Thus, fluorigenic molecules, such as the flavoenzymes whose FAD cofactors are strongly fluorescent in the oxidized state and nearly dark in the reduced state $\left(\mathrm{FADH}_{2}\right)(\mathrm{Kao}$ et al. 2008), can be used to follow single-electron transfer reaction events by measuring correlated electrochemical and spectroscopic (fluorescence) events at the single-enzyme molecule level (Zaino et al. 2015).

\section{POINT-OF-CARE DEVICES}

PoC devices are an increasingly important subject for researchers, as the need for diagnostic tools to address underserved populations grows. In response to the need for a control standard for PoC devices, particularly for use in low-resource 
and developing areas, the World Health Organization (WHO) has established criteria for the manufacture, operation, and efficacy of PoC devices via the Sexually Transmitted Diseases Diagnostics Initiative (SDI). These are known as the ASSURED criteria (Peeling et al. 2006): affordable, sensitive, specific, user-friendly, rapid and robust, equipment-free, and deliverable to end-users.

Specific values for ASSURED criteria vary based on control standards for the specific immunoassay (Yager et al. 2008). Generally, to be considered a viable PoC tool, these devices should have high sensitivity and specificity, be able to withstand storage temperatures in excess of $30^{\circ} \mathrm{C}$, and be simple enough to use that there is little need for extensive training or equipment. In addition, manufacture of $\mathrm{PoC}$ devices should be cost-effective while maintaining the test's diagnostic repeatability and reproducibility between production lots. Standards for external quality assurance, which would monitor quality and viability of the PoC tests, vary by country or region and through national or international bodies such as the European Commission or the U.S. Food and Drug Administration (Kosack et al. 2017).

Lateral flow assays (LFAs) are among the few PoC diagnostic tools that have approached ASSURED criteria, according to the WHO. LFAs commonly use either a double antibody sandwich assay or inhibitive immunoassay for analysis. The paper-based dipstick design is most common, wherein a conjugate pad contains dried reagent and a reaction membrane contains control and test lines for analyte determination. The sample mobilizes the reagentcontaining conjugate antibody (sandwich assay) or antigen (competitive assay) and signal molecule-and is wicked to the test and control lines that contain the appropriate conjugate antibodies to capture the analyte for the assay. LFAs are straightforward to use, but they are challenged with multiplex assays, typically being constrained to run multiple single assays in parallel. The most well-established LFAs are used for the detection of human chorionic gonadotropin ([hCG], the human pregnancy hormone), infectious diseases such as human immunodeficiency virus (HIV), and hepatitis $\mathrm{B}$, cardiac markers (troponin $\mathrm{C}$, creatinine kinase-muscle/brain [MB], myoglobin), and malignancies (Cheng et al. 2016).

Emerging technologies, such as imaging and image analysis, microfluidics, and nanotechnologies in PoC device design have opened new possibilities and posed new challenges (Yager et al. 2006). Advanced cameras and image analysis algorithms render LFA-based diagnostics less labor-intensive, although they require trained operators. Microfluidics allows for small sample volumes and the automation of sample preparation without a highly trained operator, and nanotechnologies allow for novel labeling techniques for easier imaging and detection. However, despite their effectiveness, designs using these advances often struggle to adhere to the low-cost manufacturing needs of end-users and commonly require external equipment operations. Paper-based microfluidic devices go some way in solving the issues of high cost and complicated operation (Yetisen et al. 2013). Multilayer paper-based microfluidic assemblies can integrate programmable valves and switches through the use of mechanical force (Martinez et al. 2010). Fluidic timing and metering can be achieved by the introduction of hydrophobic films in microchannels with $\pm 6 \%$ of total wicking time precision (Noh and Phillips 2010), while fluidic batteries have shown the ability to power a red light-emitting diode (LED) $\left(\lambda_{\mathrm{em}}=630 \mathrm{~nm}\right)$ up to $8 \mathrm{~min}$ by varying the amount of electrolyte and battery configuration with maximum short circuit current and open circuit voltage values ranging from $660 \mu \mathrm{A}$ and $1.3 \mathrm{~V}$ for one galvanic cell to $722 \mu \mathrm{A}$ and $5.0 \mathrm{~V}$ for a 16-cell battery (Thom et al. 2012). Thus, these methods have the potential to automate sample treatment and function of the device, although they have yet to be widely adopted by the medical devices industry.

\section{MICRO- AND NANO-ENABLED ELECTROPHOTONIC ASSAYS}

\section{Whole-Cell Detection}

An alternative to PCR-enabled DNA detection and identification of pathogens and infectious 
K. Fu et al.

agents relies on whole-cell detection ( $\mathrm{Hu}$ et al. 2018). A label-free and semiquantitative optical sensing platform was recently developed by our group using whole-cell mediated LSPR wavelength shifts. This versatile sensing platform has the potential to detect a broad range of potential pathogens simply by switching the analyte-specific recognition agents. Here we focus on applications to whole-cell bacterial detection, using either aptasensors or siderophore-based devices. In both biosensors, we have achieved a balance in the detection speed $(\sim 3 \mathrm{~h})$, sensitivity (one Pseudomonas aeruginosa cell and 80 Acinetobacter baumannii cells), and dynamic range (10-10 70 colony-forming units (cfu) $\mathrm{mL}^{-1}$ ), which are essentials for an ideal biosensor (Hu and Bohn 2017).

\section{Aptasensor}

Aptasensors use aptamers, which are singlestrand oligonucleotides with unique secondary and tertiary structures to bind target analytes with high affinities ( $K_{\mathrm{d}}$ from nм to $\mathrm{pm}$ ) as recognition agents (Iliuk et al. 2011). Specific aptamers are selected against their analytes (e.g., whole bacterial cells as represented by bacterial membrane proteins) through a process called systematic evolution of ligands by exponential enrichment (SELEX) (Wilson and Szostak 1999). Importantly, the affinity of aptamers toward their specific targets is comparable to that of antibody-antigen pairs. Moreover, aptamers are stable in a wide range of $\mathrm{pH}$, temperature, and buffer conditions.

In the whole-cell LSPR-based aptasensor (Hu et al. 2018), a $P$. aeruginosa-specific aptamer (Wang et al. 2011) was used to detect intact bacterial cells. The overall strategy in volves recognition of the surface-bound aptamer by a putative bacterial membrane transporter protein (Sandy and Butler 2009). Because the recognition agent (aptamer) is bound to the surface, the bacterium becomes immobilized in close proximity to the sensing volume of the LSPR elements (nanotriangles). P. aeruginosa was chosen as the model microorganism, because there is an urgent need to develop a rapid and accurate diagnostic tool to combat in- creasing antimicrobial resistance (www.who.int/ mediacentre/factsheets/fs194/en). General sensor chip design is shown in Figure 4A. The aptasensor is based on LSPR response of an hexagonal array of gold nanotriangles (Fig. 4B), which was fabricated using NSL. Sensor chips were subsequently modified with a mixture of biotinylated polyethylene glycol (Bt-PEG) thiol and PEG thiol (1:3, v:v), neutravidin, and biotinylated aptamer (Bt-aptamer) in a sandwich format. PEG thiol was used to prevent nonspecific adsorptions onto the sensor surface; Bt-PEG thiol was chosen to bind to neutravidin, which was further linked to Bt-aptamer. This carefully designed surface modification was used to efficiently capture whole-cell $P$. aeruginosa. After careful processing, with special attention paid to removing unwanted agents (e.g., salts, nonspecifically bound macromolecules, etc.), and to preventing bacterial cell lysis, the bacteria containing sensor chips (Fig. 4C) were gently washed, dried, and characterized in a UVvisible spectrometer with an integrating sphere. The whole-cell LSPR aptasensor exhibits an astonishing sensitivity, down to a single bacterial cell, with a linear range from 1 to $1000 \mathrm{cfu} \mathrm{mL}^{-1}$, as well as a clinically relevant detection range of $10-10^{5} \mathrm{cfu} \mathrm{mL}^{-1}$. Additionally, this aptasensor is selective for $P$. aeruginosa strain PAO1 over strain PA14, Escherichia coli, and Staphylococcus aureus. Currently, the detection time is limited to $\sim 3 \mathrm{~h}$ by the need for careful inactivation of live bacteria. However, the detection time can be reduced to $\sim 1$ h or less in clinical settings. In addition, the LSPR aptasensor chips were shown to be stable in ambient conditions for over 2 months, raising the possibility of their use in hostile climates, such as those found in many resource-limited areas.

\section{Siderophore-Based Devices}

To demonstrate the versatility of the whole-cell bacterial-sensing platform, the same sensor chip and basic surface modifications are being used in a siderophore-based sensor. Siderophores are a class of small (500-1500 Da) molecules synthesized by bacteria, fungi, and plants to acquire ferric iron $\left(\mathrm{Fe}^{3+}\right)$ with high affinity $\left(K_{\mathrm{f}}>10^{30}\right)$ 

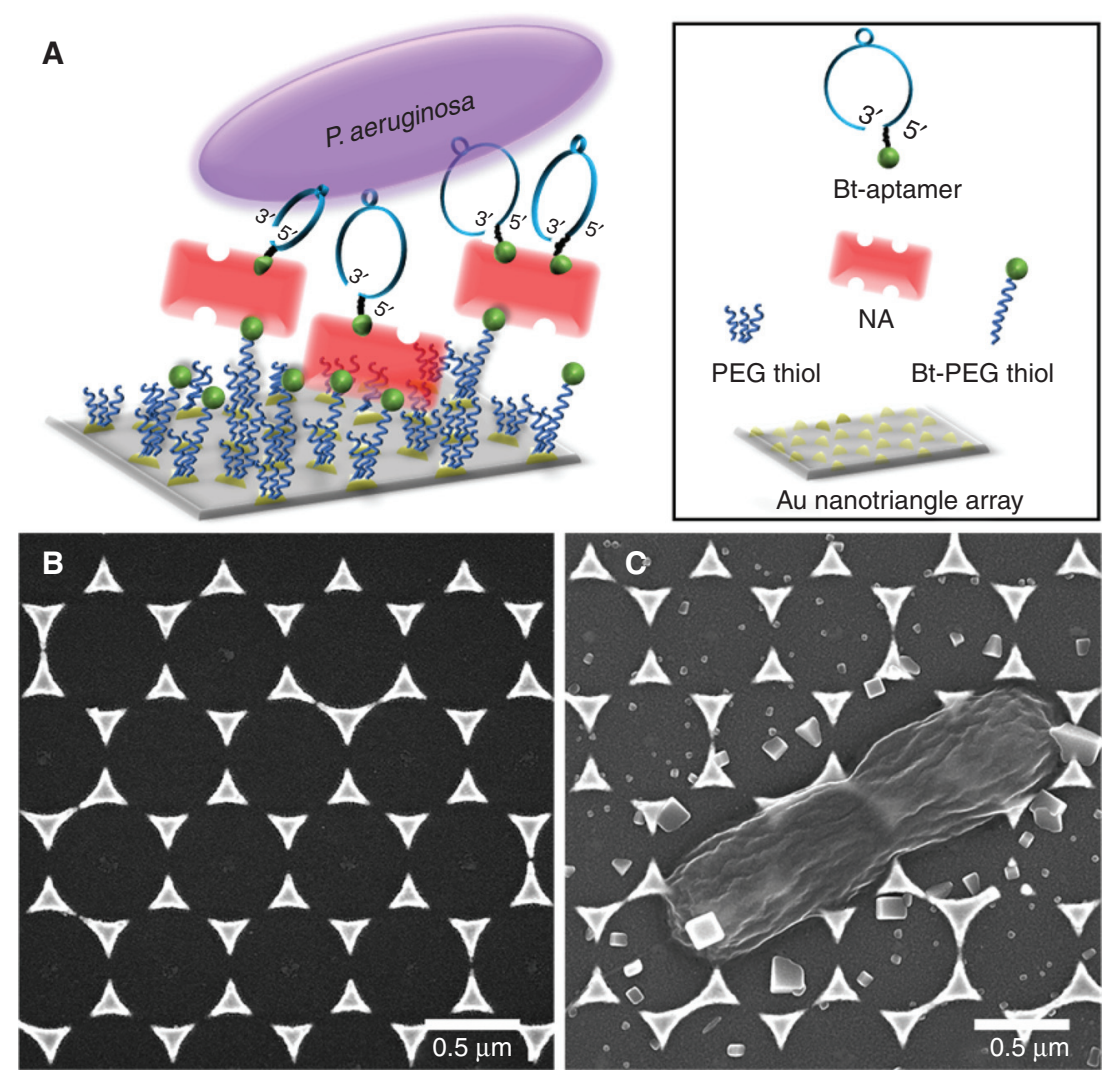

Figure 4. Localized surface plasmon resonance (LSPR) sensors are sensitive, but selectivity has to be added by constructing the device to present a molecular recognition motif, in this case an aptamer specific for Pseudomonas aeruginosa, on the surface. (A) Schematic illustration of the mechanism of bacterial recognition and pulldown used in the aptasensor. Bt, Biotin; NA, neutravidin; PEG, poly(ethylene glycol). Representative scanning electron microscope images of Au nanotriangle array $(B)$ and bacterial cell(s) of $P$. aeruginosa strain PAO1 captured on a modified sensor surface $(C)$. (From Hu et al. 2018; adapted, with permission, from the American Chemical Society (C) 2018.)

from the environment (Hider and Kong 2010). Because of the low availability of free $\mathrm{Fe}^{3+}$ in the environment $\left(10^{-9}\right.$ to $\left.10^{-18} \mathrm{M}\right)$ as well as in se$\operatorname{rum}\left(10^{-24} \mathrm{M}\right)$, and the fact that $\mathrm{Fe}^{3+}$ is essential for key biological processes in almost all microorganisms, plants, and animals, microbes and plants produce siderophores to chelate $\mathrm{Fe}^{3+}$ when the intracellular iron concentration is below $\sim 10^{-6}$ M (Miethke and Marahiel 2007). More than 500 siderophores have been discovered, of which 270 structures have been identified, falling into three main classes of siderophores: catecholates, hydroxamates, and ( $\alpha$-hydroxy) carboxylates based on the iron- binding moieties (Hider and Kong 2010). "Mixed-type" siderophores have also been identified. Different siderophores exhibit various affinities toward $\mathrm{Fe}^{3+}$, ranging over $\sim 30$ orders of magnitude. $\mathrm{Fe}^{3+}$ scavenged from the environment (in the form of siderophore- $\mathrm{Fe}^{3+} \mathrm{com}$ plex) is then recognized and actively transported through the microbial cell membrane into the cytoplasm with the assistance of various receptor, binding, and transport proteins (Sandy and Butler 2009).

In the siderophore-based LSPR biosensor being developed by our group, we use a laboratory-synthesized siderophore, specifically a bis- 
K. Fu et al.

catecholate-monohydroxamate mixed ligand siderophore with three polyethylene glycol repeating units linked to a biotin, as a recognition agent to target $A$. baumannii, a top priority pathogen by the WHO in 2017 (www.who.int/ mediacentre/news/releases/2017/bacteria-antibi otics-needed/en). Specifically, NSL-fabricated $\mathrm{Au}$ nanotriangle arrays were modified with Bt-PEG thiol/PEG thiol (1:3, v:v), neutravidin, Bt-siderophore, and $\mathrm{Fe}^{3+}$ acetylacetonate. A. baumannii was added and incubated, allowing the bacteria to recognize and bind to the Bt-siderophore- $\mathrm{Fe}^{3+}$ complexes on the sensor surface. Bacteria attempt to actively transport the complex through their cell membranes, but given that the Bt-siderophore- $\mathrm{Fe}^{3+}$ complexes are anchored onto the sensor surface the result is that the A. baumannii is pulled from solution and immobilized. Initial work on the LSPR siderophore-based sensor has yielded promising results. LSPR response has shown that the siderophore-based biosensor can recognize and detect A. baumannii down to $400 \mathrm{cfu} \mathrm{mL}^{-1}$ (equivalent to 80 cells), with a dynamic range from $0.4 \times 10^{3}$ to $0.4 \times 10^{7} \mathrm{cfu} \mathrm{mL}^{-1}$. The decreased sensitivity compared to the aptasensor can be explained by (1) impurities in the synthesized Bt-siderophore, and (2) the inherent affinity of this siderophore recognition system. Additionally, the selectivity and longevity of this siderophore-based biosensor are currently under investigation.

\section{Molecular Biomarker Detection \\ Closed Bipolar Electrochemical Assays}

Taking advantage of CBE structures opens up new approaches for electrochemical assays. Redox reactions at the poles (driving electrodes) of the BPE enable electron transfer throughout the entire system, so species with redox activity can be detected straightforwardly within a $\mathrm{CBE}$ configuration. CBE systems were initially used for voltammetric studies, in which electrochemical reactions on bipolar microelectrodes and nanoelectrodes were coupled in a CBE architecture and were shown to exhibit different voltammetric responses (Cox et al. 2012; Guerrette et al.
2012). Taking advantage of fluorigenic redox reactions as reporters, they also introduced fluorescence-enabled electrochemical microscopy (FEEM) for imaging transient and heterogeneous electrochemical processes by coupling fluorescence microscopy to $\mathrm{CBE}$ structures (Oja and Zhang 2014). This technique enables the use of fluorescence microscopy to observe conventional electrochemical processes on large electrochemical arrays with proper choice of fluorescence probes. Wang and colleagues integrated microfluidics with the CBE system to develop a dual-channel mode electrochemiluminescence (ECL)-sensing microchip (Luo et al. 2013). In their serial CBE configuration, voltage drop across the ECL reporting cell changed as a function of the conductivity in the analyte cell, thus establishing a quantitative relation between ECL response and the sample conductivity (Zhang et al. 2016c). The use of a serial $\mathrm{CBE}$ structure also enables all the oxidants, reductants, or related chemical probes to be determined in different reservoirs in a single device (Zhang et al. 2014). CBEs constructed from interdigitated electrode arrays (IDEAs), which support the coupling of independent redox reactions in a CBE structure, were developed by our group. When coupled to fluorescence imaging, this approach allows a diverse set of spatially varying electrochemical phenomena to be explored (Xu et al. 2016b). Extending this idea, rapid, portable, and cost-effective measurement of indicator absorption was used to implement a CBE-enabled method for electrochemical sensing of redox-active biomarkers based on the electrochromic response of MV reporter and rendered in a field-deployable format ( $\mathrm{Xu}$ et al. 2016a). This method produced a linear relationship among the analyte concentration, the quantity of MV generated, and the colorimetric response, yielding a limit of detection (LOD) of $1.0 \mu \mathrm{M}$. Electrochemical detection has also been performed in a split BPE design, by coupling the electrochemical cell with an LED as a signal readout to provide a low-cost analytical tool with high throughput for portable analysis (Zhang et al. 2015). In addition to sensing applications, BPE structures have also been used for electrocatalyst screening (Zhang et al. 2016b). 


\section{Indirect CBE Assays}

In the context of diagnostic assays, we define "indirect" as those redox reactions that happen in the analytical cell with the assistance of a redox mediator and/or redox enzyme. As mentioned above, model redox-active analytes like ferricyanide and ferrocene have been used in model CBE-sensing assays (Xu et al. 2016a). To extend the CBE-sensing assays to general biosensing applications and recognizing that many biologically relevant analytes (e.g., metabolites, such as glucose, lactate, and uric acid) are not redox-active, it is imperative to develop indirect redox processes that can be used in place of direct CBE assays. For biomarkers that are not redox-active, electrochemical mediators can be employed to couple enzymatic recognition reactions to the electrode as specific mediator-electrocatalyst pairs (Xu et al. 2017).

The detection of glucose has been used to show the working principle of indirect $\mathrm{CBE}$ assays. Glucose, which is not directly redox-active, was mixed with glucose oxidase (enzyme) and ferricyanide (mediator), and then detection was accomplished by the enzyme-catalyzed oxidation of glucose to produce gluconic acid, converting the glucose oxidase back to its reduced form. Reduced glucose oxidase then reacts with ferricyanide to regenerate oxidized glucose oxidase and ferrocyanide. To complete the redox cycle, a sufficiently oxidizing potential is applied to working electrode to convert ferrocyanide back to ferricyanide, the resulting current either being measured directly in an amperometric assay or coupled to an MV-containing reporter cell to develop an electrochromic response. Owing to the regeneration of glucose oxidase (oxidized form) and ferricyanide, the reaction continues as long as glucose is present in the mixture. For glucose, a linear response was observed up to $1 \mathrm{~mm}$ with an LOD of $\sim 180 \mu \mathrm{m}$. More importantly, the scheme outlined here is general for non-redox-active analytes that can react with the aid of an oxidoreductase enzyme and electrochemical mediators. As an example, LODs of 110 and $180 \mu \mathrm{m}$ were obtained for uric acid and lactate, respectively, both of which are sufficient for routine clinical monitoring.
To extend the capability of device for multiplex operation, the indirect CBE assay has been modified by integrating multiple (three) sets of detection chemistries into a single device to accomplish the simultaneous determination of glucose, lactate, and uric acid, as shown in Figure 5. This indirect CBE assay was used to simultaneously detect $2.5 \mathrm{~mm}$ each of glucose, lactate, and uric acid. An obvious purple color was observed in the presence of analytes, confirming that multiple analytes can be determined simultaneously and independently by color change in a single multiplex device. Further enhancing the utility and $\mathrm{PoC}$ readiness of the device, the analytical cell was fabricated as a disposable, paper-based carbon electrode without any pretreatment. Importantly, both the analytical cell and the reporter cell can be optimized separately. For example, the sensitivity of biomarker detection can be tuned by using reporter cells with different volumes. Because electrochemical reactions are quantitative coulometrically, the number of absorbers $\left(\mathrm{MV}^{+\bullet}\right)$ generated is proportional to analyte concentration, but the absorbance (color change) in the reporter cell is proportional to $\mathrm{MV}^{+\bullet}$ concentration. Thus, decreasing the reporter cell volume enhances the sensitivity of the measurement, demonstrating the potential of $\mathrm{CBE}$ to test biomarkers with different detection ranges.

\section{Other (Future) Biomarkers}

Besides the above metabolites, other biomarkers can be detected with the CBE strategy. Typical protocols rely on efficient modification of the working electrode with a biomolecular recognition agent, which recognizes and captures the target analyte, producing an electrochemical signal that is read out (Kimmel et al. 2012; Turner 2013; Labib et al. 2016). Numerous biomarkers, including proteins $(\mathrm{Wu}$ et al. 2013), DNA/RNA molecules (Wu et al. 2012), and pathogens (Liu et al. 2016a), have been successfully detected by CBE assays. Zhang and coworkers demonstrated fluorescenceenabled CBE assays, which has proven to be capable of recording the transient extracellular vesicle release of neurotransmitter molecules 
K. Fu et al.

A
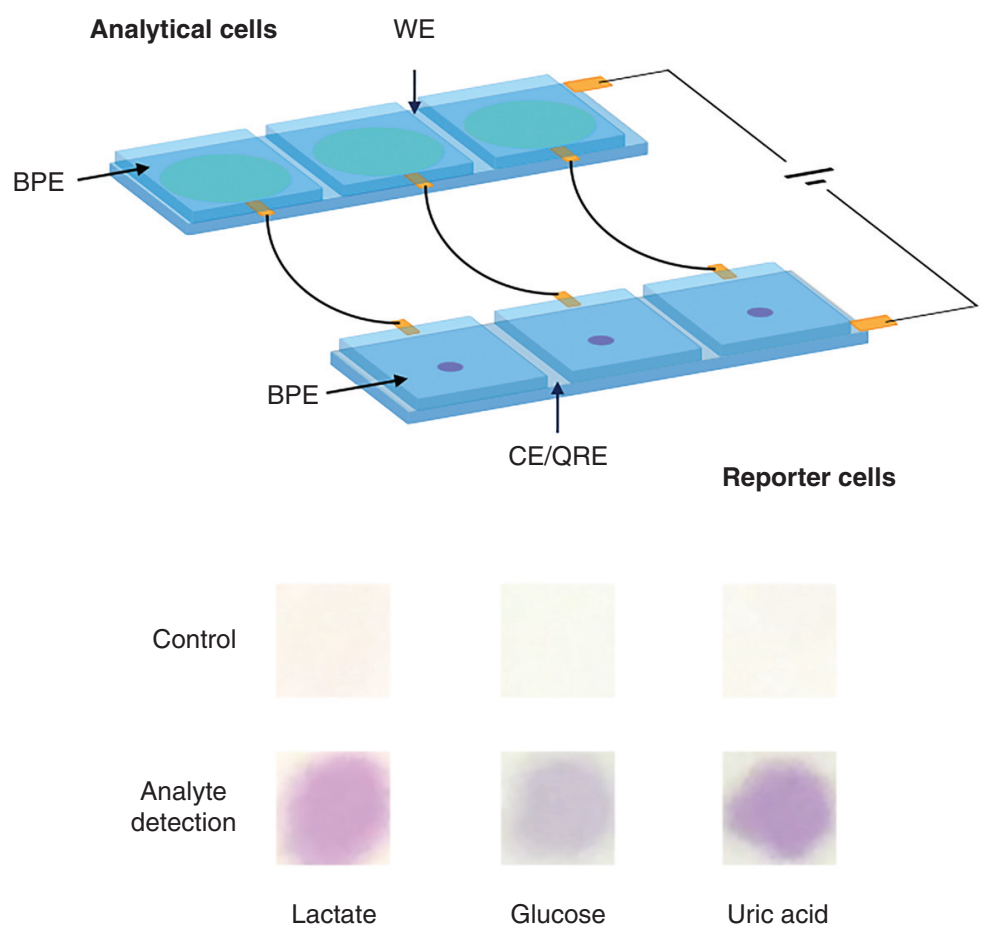

Figure 5. Realistic point-of-care devices for early diagnosis of sepsis conditions will require the simultaneous multiplex detection (binary classification) of multiple biomarkers. (A) Schematic illustration of the closed bipolar electrode (CBE)-enabled electrochromic sensor architecture for multiple analyte detection. The analytical cell is composed of closely spaced $(\sim 30 \mu \mathrm{m})$ indium tin oxide (ITO) and platinum (Pt) planar electrodes, with the Pt serving as one half of a CBE. The reporter cell is constructed with two ITO planar electrodes similarly spaced. The close spacing of the electrodes enables redox cycling, which enhances the signal. (B) Color change in reporter cells for simultaneous detection of $2.5 \mathrm{~mm}$ lactate, $2.5 \mathrm{~mm}$ glucose, and $2.5 \mathrm{~mm}$ uric acid. Control samples were run without the specific analyte but with all other components (enzyme, $\mathrm{Fe}(\mathrm{CN})_{6}{ }^{3-/ 4-}$ mediator) present. BPE, Bipolar electrode; WE, working electrode; CE, counter electrode; QRE, quasi-reference electrode. (From Xu et al. 2017; adapted, with permission, from the authors.)

(e.g., dopamine) (Cox et al. 2012; Oja Stephen and Zhang 2015). Xu and coworkers used ECLbased CBEs to detect several important biomarkers, including HL-60 tumor cells (Zhang et al. 2016a), and prostate-specific antigen (Wu et al. 2015).

Recently, our laboratory has begun to develop integrated CBE-based biosensing platforms aimed at the early and accurate diagnosis of sepsis, a potentially life-threatening syndrome caused by infection. Several biomarkers are used in clinical tests to diagnose and manage the severity of patient's illness (e.g., lactate, Creactive protein, the liver enzymes ALT and AST, creatinine, procalcitonin [PCT], and bili- rubin) (Reinhart et al. 2012). There is hope that a multiplex assay ported to a PoC device, by reducing the time to accurate diagnosis, can have a substantive impact on patient outcomes. It is a challenging task to detect multiple analytes simultaneously in a portable device. For example, there are enormous concentration differences exhibited by the diagnostic biomarkers in the blood of patients (e.g., lactate and PCT are millimolar and nanomolar, respectively), thus making multiple analyte detection (much less quantitation) within one device extremely complicated. However, the combination of relevant enzyme chemistries and indirect CBE assays, gives impetus to our efforts to solve these prob- 
lems, and preliminary results indicate that CBE assays have the potential to screen phenotypes that require accurate multiple-biomarker assays in a PoC format.

\section{CONCLUSIONS}

The inclusion of microscale and nanoscale construction principles into electrophotonic devices has generated a new set of diagnostic devices with improved performance characteristics relative to existing PoC approaches. These diagnostics exploit the capacity of small-scale metaldielectric assemblies to manipulate electromagnetic radiation and electron transfer reactions simultaneously, making it possible to couple electron transfer events to optical emission, absorption, and scattering readouts, thereby creating new ways to assay for pathogens from the molecular scale up to whole cells. Thus, we continue to look to advances in the previously separate fields of high sensitivity analytical electrochemistry and nanophotonics for exciting new opportunities to be exploited.

\section{ACKNOWLEDGMENTS}

Work described here, which was carried out in the authors' laboratory, was made possible by support from the National Institute for Allergies and Infectious Diseases (R01AI113219-01), the National Science Foundation (NSF14047440), and the Department of Energy (DE FG02 07ER15851).

\section{REFERENCES}

Bertok T, Klukova L, Sediva A, Kasák P, Semak V, Micusik M, Omastova M, Chovanová L, Vlček M, Imrich R, et al. 2013. Ultrasensitive impedimetric lectin biosensors with efficient antifouling properties applied in glycoprofiling of human serum samples. Analyt Chem 85: 7324-7332.

Bundy J, Fenselau C. 1999. Lectin-based affinity capture for MALDI-MS analysis of bacteria. Analyt Chem 71: 1460-1463.

Byers JC, Nadappuram BP, Perry D, McKelvey K, Colburn AW, Unwin PR. 2015. Single molecule electrochemical detection in aqueous solutions and ionic liquids. Analyt Chem 87: 10450-10456.

Cai Z, Sasmal A, Liu XW, Asher SA. 2017. Responsive photonic crystal carbohydrate hydrogel sensor materials for selective and sensitive lectin protein detection. ACS Sensors 2: 1474-1481.

Chang YF, Wang WH, Hong YW, Yuan RY, Chen KH, Huang YW, Lu PL, Chen YH, Chen YMA, Su LC, et al. 2018. Simple strategy for rapid and sensitive detection of avian influenza A H7N9 virus based on intensitymodulated SPR biosensor and new generated antibody. Analyt Chem 90: 1861-1869.

Cheng CMK, Kuan CM, Chen J. 2016. In-vitro diagnostic devices: Introduction to current point-of-care diagnostic devices. Springer, Basel, Switzerland.

Chow KF, Mavre F, Crooks JA, Chang BY, Crooks RM. 2009. A large-scale, wireless electrochemical bipolar electrode microarray. J Am Chem Soc 131: 8364-8365.

Cox JT, Guerrette JP, Zhang B. 2012. Steady-state voltammetry of a microelectrode in a closed bipolar cell. Analyt Chem 84: 8797-8804.

Delehanty JB, Ligler FS. 2002. A microarray immunoassay for simultaneous detection of proteins and bacteria. Analyt Chem 74: 5681-5687.

Dong S, Zhao R, Zhu J, Lu X, Li Y, Qiu S, Jia L, Jiao X, Song S, Fan C, et al. 2015. Electrochemical DNA biosensor based on a tetrahedral nanostructure probe for the detection of avian influenza A (H7N9) virus. ACS Appl Mater Interf 7: 8834-8842.

Doorneweerd DD, Henne WA, Reifenberger RG, Low PS. 2010. Selective capture and identification of pathogenic bacteria using an immobilized siderophore. Langmuir 26: 15424-15429.

Eid J, Fehr A, Gray J, Luong K, Lyle J, Otto G, Peluso P, Rank D, Baybayan P, Bettman B, et al. 2009. Real-time DNA sequencing from single polymerase molecules. Science 323: $133-138$.

Elnifro EM, Ashshi AM, Cooper RJ, Klapper PE. 2000. Multiplex PCR: Optimization and application in diagnostic virology. Clin Microbiol Rev 13: 559-570.

Fosdick SE, Crooks RM. 2012. Bipolar electrodes for rapid screening of electrocatalysts. J Am Chem Soc 134: 863-866.

Fosdick SE, Knust KN, Scida K, Crooks RM. 2013. Bipolar electrochemistry. Angew Chem Int Ed 52: 10438-10456.

Fratamico PM, Strobaugh TP, Medina MB, Gehring AG. 1998. Detection of Escherichia coli O157: H7 using a surface plasmon resonance biosensor. Biotechnol Tech 12: 571-576.

Fu K, Han D, Ma C, Bohn PW. 2016. Electrochemistry at single molecule occupancy in nanopore-confined recessed ring-disk electrode arrays. Faraday Discuss 193: 51-64.

Gau JJ, Lan EH, Dunn B, Ho CM, Woo JCS. 2001. A MEMS based amperometric detector for E. coli bacteria using selfassembled monolayers. Biosens Bioelectron 16: 745-755.

Grow AE, Wood LL, Claycomb JL, Thompson PA. 2003. New biochip technology for label-free detection of pathogens and their toxins. J Microbiol Methods 53: 221-233.

Guerrette JP, Oja SM, Zhang B. 2012. Coupled electrochemical reactions at bipolar microelectrodes and nanoelectrodes. Analyt Chem 84: 1609-1616.

Haes AJ, Van Duyne RP. 2002. A nanoscale optical biosensor: Sensitivity and selectivity of an approach based on the localized surface plasmon resonance spectroscopy of triangular silver nanoparticles. J Am Chem Soc 124: 10596-10604.

Haes AJ, Van Duyne RP. 2004. Preliminary studies and potential applications of localized surface plasmon reso- 
K. Fu et al.

nance spectroscopy in medical diagnostics. Exp Rev Med Diagn 4: 527-537.

Hall WP, Modica J, Anker J, Lin Y, Mrksich M, Van Duyne RP. 2011a. A conformation- and ion-sensitive plasmonic biosensor. Nano Lett 11: 1098-1105.

Hall WP, Ngatia SN, Van Duyne RP. 2011b. LSPR biosensor signal enhancement using nanoparticle-antibody conjugates. J Phys Chem C 115: 1410-1414.

Hall AS, Friesen SA, Mallouk TE. 2013. Wafer-scale fabrication of plasmonic crystals from patterned silicon templates prepared by nanosphere lithography. Nano Lett 13: 2623-2627.

Hendry E, Carpy T, Johnston J, Popland M, Mikhaylovskiy RV, Lapthorn AJ, Kelly SM, Barron LD, Gadegaard N, Kadodwala M. 2010. Ultrasensitive detection and characterization of biomolecules using superchiral fields. Nat Nanotechn 5: 783-787.

Hider RC, Kong X. 2010. Chemistry and biology of siderophores. Nat Prod Rep 27: 637-657.

Hoeben FJM, Meijer FS, Dekker C, Albracht SPJ, Heering HA, Lemay SG. 2008. Toward single-enzyme molecule electrochemistry: NiFe-hydrogenase protein film voltammetry at nanoelectrodes. ACS Nano 2: 2497-2504.

Hu J, Bohn PW. 2017. Optical biosensing of bacteria and bacterial communities. J Anal Test 1: 4.

Hu J, Fu K, Bohn PW. 2018. Whole-cell Pseudomonas aeruginosa localized surface plasmon resonance aptasensor. Analyt Chem 90: 2326-2332.

Hulteen JC, Van Duyne RP. 1995. Nanosphere lithography: A materials general fabrication process for periodic particle array surfaces. J Vac Sci Technol A 13: 1553-1558.

Iliuk AB, Hu L, Tao WA. 2011. Aptamer in bioanalytical applications. Analyt Chem 83: 4440-4452.

Jung K, Fleischhacker M, Rabien A. 2010. Cell-free DNA in the blood as a solid tumor biomarker-A critical appraisal of the literature. Clin Chim Acta 411: 1611-1624.

Kao YT, Saxena C, He TF, Guo LJ, Wang LJ, Sancar A, Zhong DP. 2008. Ultrafast dynamics of flavins in five redox states. J Am Chem Soc 130: 13132-13139.

Kimmel DW, LeBlanc G, Meschievitz ME, Cliffel DE. 2012. Electrochemical sensors and biosensors. Analyt Chem 84: 685-707.

Kosack CS, Page AL, Klaster PR. 2017. A guide to aid the selection of diagnostic tests. In Policy and practice, pp. 639-645. Bulletin of the World Health Organization, World Health Organization, Geneva, Switzerland.

Labib M, Sargent EH, Kelley SO. 2016. Electrochemical methods for the analysis of clinically relevant biomolecules. Chem Rev 116: 9001-9090.

Levene MJ, Korlach J, Turner SW, Foquet M, Craighead HG Webb WW. 2003. Zero-mode waveguides for singlemolecule analysis at high concentrations. Science 299: 682-686.

Liu H, Crooks RM. 2012. Paper-based electrochemical sensing platform with integral battery and electrochromic read-out. Analyt Chem 84: 2528-2532.

Liu H, Zhou X, Liu W, Yang X, Xing D. 2016a. Paper-based bipolar electrode electrochemiluminescence switch for label-free and sensitive genetic detection of pathogenic bacteria. Analyt Chem 88: 10191-10197.
Liu M, Liu R, Wang D, Liu CL, Zhang CS. 2016b. A low-cost, ultraflexible cloth-based microfluidic device for wireless electrochemiluminescence application. Lab Chip 16: 2860-2870.

Loget G, Zigah D, Bouffier L, Sojic N, Kuhn A. 2013. Bipolar electrochemistry: From materials science to motion and beyond. Acc Chem Res 46: 2513-2523.

Lu HP, Xie XS. 1999. Single-molecule spectroscopy studies of molecular dynamics in chemical and biological systems. Z Phys Chem 212: 59-66.

Luo YX, Rasmussen JO, Hamilton JH, Ramayya AV, Frauendorf S, Hwang JK, Stone NJ, Zhu SJ, Brewer NT, Wang E et al. 2013. New insights into the nuclear structure in neutron-rich Pd. Nucl Phys A 919: 67-98.

Ma C, Zaino LP, Bohn PW. 2015. Self-induced redox cycling coupled luminescence on nanopore recessed disk-multiscale bipolar electrodes. Chem Sci 6: 3173-3179.

Mannoor MS, Zhang S, Link AJ, McAlpine MC. 2010. Electrical detection of pathogenic bacteria via immobilized antimicrobial peptides. Proc Natl Acad Sci 107: 1920719212.

Martinez AW, Phillips ST, Nie Z, Cheng CM, Carrilho E, Wiley BJ, Whitesides GM. 2010. Programmable diagnostic devices made from paper and tape. Lab Chip 10: 24992504.

Mavre F, Anand RK, Laws DR, Chow KF, Chang BY, Crooks JA, Crooks RM. 2010. Bipolar electrodes: A useful tool for concentration, separation, and detection of analytes in microelectrochemical systems. Analyt Chem 82: 8766-8774.

Miethke M, Marahiel MA. 2007. Siderophore-based iron acquisition and pathogen control. Microbiol Mol Biol Rev 71: 413-451.

Muguruma H, Hoshino T, Matsui Y. 2011. Enzyme biosensor based on plasma-polymerized film-covered carbon nanotube layer grown directly on a flat substrate. ACS Appl Mater Interf 3: 2445-2450.

Nidzworski D, Siuzdak K, Niedziałkowski P, Bogdanowicz R, Sobaszek M, Ryl J, Weiher P, Sawczak M, Wnuk E, Goddard WA, et al. 2017. A rapid-response ultrasensitive biosensor for influenza virus detection using antibody modified boron-doped diamond. Sci Rep 7: 15707.

Noh H, Phillips ST. 2010. Metering the capillary-driven flow of fluids in paper-based microfluidic devices. Anal Chem 82: 4181-4187.

Oja SM, Zhang B. 2014. Imaging transient formation of diffusion layers with fluorescence-enabled electrochemical microscopy. Analyt Chem 86: 12299-12307.

Oja SM, Zhang B. 2015. Electrogenerated chemiluminescence reporting on closed bipolar microelectrodes and the influence of electrode size. ChemElectroChem 3: 457-464.

Peeling RW, Holmes KK, Mabey D, Ronald A. 2006. Rapid tests for sexually transmitted infections (STIs): The way forward. Sex Transm Infect 82: v1-v6.

Perdue RK, Laws DR, Hlushkou D, Tallarek U, Crooks RM. 2009. Bipolar electrode focusing: The effect of current and electric field on concentration enrichment. Analyt Chem 81: 10149-10155.

Pestov D, Wang X, Ariunbold GO, Murawski RK, Sautenkov VA, Dogariu A, Sokolov AV, Scully MO. 2008. Single-shot detection of bacterial endospores via coherent Raman spectroscopy. Proc Natl Acad Sci 105: 422-427. 
Reinhart K, Bauer M, Riedemann NC, Hartog CS. 2012. New approaches to sepsis: Molecular diagnostics and biomarkers. Clin Microbiol Rev 25: 609-634.

Rowe-Taitt CA, Golden JP, Feldstein MJ, Cras JJ, Hoffman KE, Ligler FS. 2000. Array biosensor for detection of biohazards. Biosens Bioelectron 14: 785-794.

Sandy M, Butler A. 2009. Microbial iron acquisition: Marine and terrestrial siderophores. Chem Rev 109: 4580-4595.

Sekretaryova AN, Beni V, Eriksson M, Karyakin AA, Turner APF, Vagin MY. 2014. Cholesterol self-powered biosensor. Analyt Chem 86: 9540-9547.

Shendure J, Ji HL. 2008. Next-generation DNA sequencing. Nat Biotechnol 26: 1135-1145.

Shinohara S, Tanaka D, Okamoto K, Tamada K. 2015. Colorimetric plasmon sensors with multilayered metallic nanoparticle sheets. Phys Chem Chem Phys 17: 18606-18612.

Taylor AD, Ladd J, Yu Q, Chen S, Homola J, Jiang S. 2006 Quantitative and simultaneous detection of four foodborne bacterial pathogens with a multi-channel SPR sensor. Biosens Bioelectron 22: 752-758.

Thom NK, Yeung K, Pillion MB, Phillips ST. 2012. "Fluidic batteries" as low-cost sources of power in paper-based microfluidic devices. Lab Chip 12: 1768-1770.

Tripathi SM, Bock WJ, Mikulic P, Chinnappan R, Ng A Tolba M, Zourob M. 2012. Long period grating based biosensor for the detection of Escherichia coli bacteria. Biosens Bioelectron 35: 308-312.

Turner APF. 2013. Biosensors: Sense and sensibility. Chem Soc Rev 42: 3184-3196.

Ulrich C, Andersson O, Nyholm L, Bjorefors F. 2008 Formation of molecular gradients on bipolar electrodes. Angew Chem Int Ed 47: 3034-3036.

Urmann K, Arshavsky-Graham S, Walter JG, Scheper T, Segal E. 2016. Whole-cell detection of live Lactobacillus acidophilus on aptamer-decorated porous silicon biosensors. Analyst 141: 5432-5440.

Varshney M, Li YB, Srinivasan B, Tung S. 2007. A label-free, microfluidics and interdigitated array microelectrodebased impedance biosensor in combination with nanoparticles immunoseparation for detection of Escherichia coli O157:H7 in food samples. Sens Actuat B 128: 99-107.

Wang Y, Hernandez RM, Bartlett DJ, Bingham JM, Kline TR, Sen A, Mallouk TE. 2006. Bipolar electrochemical mechanism for the propulsion of catalytic nanomotors in hydrogen peroxide solutions. Langmuir 22: 10451-10456.

Wang KY, Zeng YL, Yang XY, Li WB, Lan XP. 2011. Utility of aptamer-fluorescence in situ hybridization for rapid detection of Pseudomonas aeruginosa. Eur J Clin Microbiol Infect Dis 30: 273-278.

Watts HJ, Lowe CR, Pollardknight DV. 1994. Optical biosensor for monitoring microbial cells. Analyt Chem 66 2465-2470.

Willets KA, Van Duyne RP. 2007. Localized surface plasmon resonance spectroscopy and sensing. Annu Rev Phys Chem 58: 267-297.

Wilson DS, Szostak JW. 1999. In vitro selection of functional nucleic acids. Annu Rev Biochem 68: 611-647.

Wu MS, Qian Gs, Xu JJ, Chen HY. 2012. Sensitive electrochemiluminescence detection of c-Myc mRNA in breast cancer cells on a wireless bipolar electrode. Analyt Chem 84: 5407-5414.
Wu MS, Yuan DJ, Xu JJ, Chen HY. 2013. Sensitive electrochemiluminescence biosensor based on Au-ITO hybrid bipolar electrode amplification system for cell surface protein detection. Analyt Chem 85: 11960-11965.

Wu MS, Liu Z, Shi HW, Chen HY, Xu JJ. 2015. Visual electrochemiluminescence detection of cancer biomarkers on a closed bipolar electrode array Chip. Analyt Chem 87: 530-537.

Xu W, Fu KY, Ma CX, Bohn PW. 2016a. Closed bipolar electrode-enabled dual-cell electrochromic detectors for chemical sensing. Analyst 141: 6018-6024.

Xu W, Ma CX, Bohn PW. 2016b. Coupling of independent electrochemical reactions and fluorescence at closed bipolar interdigitated electrode arrays. ChemElectroChem 3: $422-428$.

Xu W, Fu K, Bohn PW. 2017. Electrochromic sensor for multiplex detection of metabolites enabled by closed bipolar electrode coupling. ACS Sensors 2: 1020-1026.

Yager P, Edwards T, Fu E, Helton K, Nelson K, Tam MR, Weigl BH. 2006. Microfluidic diagnostic technologies for global public health. Nature 442: 412-418.

Yager P, Domingo GJ, Gerdes J. 2008. Point-of-care diagnostics for global health. Annu Rev Biomed Eng 10: 107-144.

Yetisen AK, Akram MS, Lowe CR. 2013. Paper-based microfluidic point-of-care diagnostic devices. Lab Chip 13: 2210-2251.

Zaino LPI, Grismer DA, Han D, Crouch GM, Bohn PW. 2015. Single molecule spectroelectrochemistry of freely diffusing flavin mononucleotide in zero-dimensional nanophotonic structures. Faraday Disc 184: 101-115.

Zevenbergen MAG, Krapf D, Zuiddam MR, Lemay SG. 2007. Mesoscopic concentration fluctuations in a fluidic nanocavity detected by redox cycling. Nano Lett 7: 384388.

Zevenbergen M, Singh P, Goluch ED, Wolfrum B, Lemay S. 2009. Electrochemical correlation spectroscopy in nanofluidic cavities. Anal Chem 81: 8203-8212.

Zhang XW, Li J, Jia XF, Li DY, Wang EK. 2014. Full-featured electrochemiluminescence sensing platform based on the multichannel closed bipolar system. Analyt Chem 86: 5595-5599.

Zhang XW, Chen CG, Yin JY, Han YC, Li J, Wang EK. 2015. Portable and visual electrochemical sensor based on the bipolar light emitting diode electrode. Analyt Chem 87: 4612-4616.

Zhang HR, Wang YZ, Zhao W, Xu JJ, Chen HY. 2016a. Visual color-switch electrochemiluminescence biosensing of cancer cell based on multichannel bipolar electrode Chip. Analyt Chem 88: 2884-2890.

Zhang XW, Shang CS, Gu WL, Xia Y, Li J, Wang E. 2016b. A renewable display platform based on the bipolar electrochromic electrode. ChemElectroChem 3: 383-386.

Zhang XW, Zhai QF, Xu L, Li J, Wang EK. 2016c. Paperbased electrochemiluminescence bipolar conductivity sensing mechanism: A critical supplement for the bipolar system. J Electroanal Chem 781: 15-19.

Zhang X, Zhai Q, Xing H, Li J, Wang E. 2017. Bipolar electrodes with $100 \%$ current efficiency for sensors. ACS Sensors 2: 320-326. 


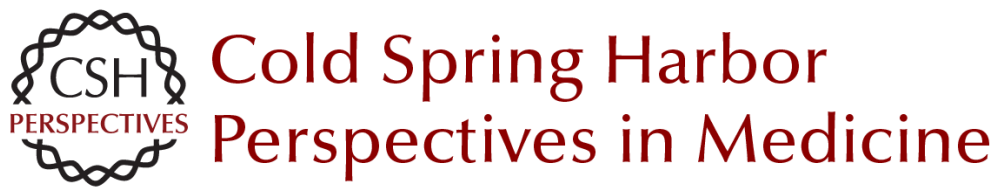

\section{Microscale and Nanoscale Electrophotonic Diagnostic Devices}

Kaiyu Fu, Wei Xu, Jiayun Hu, Arielle Lopez and Paul W. Bohn

Cold Spring Harb Perspect Med 2019; doi: 10.1101/cshperspect.a034249 originally published online August 13, 2018

\section{Subject Collection Bioelectronic Medicine}

Neural Control of Inflammation: Bioelectronic Medicine in Treatment of Chronic Inflammatory

Disease

Michael Eberhardson, Laura Tarnawski, Monica Centa, et al.

Noninvasive Neuromodulation of Peripheral Nerve Pathways Using Ultrasound and Its Current Therapeutic Implications Christopher Puleo and Victoria Cotero

Enteric Neuromodulation for the Gut and Beyond Yogi A. Patel and Pankaj J. Pasricha

\section{Optogenetic Control of the Peripheral Nervous} System

\author{
Rui B. Chang
}

Closed-Loop Neuromodulation in Physiological and Translational Research Stavros Zanos

Electrical Impedance Methods in Neuromuscular Assessment: An Overview Seward B. Rutkove and Benjamin Sanchez

Optogenetic Medicine: Synthetic Therapeutic

Solutions Precision-Guided by Light Haifeng Ye and Martin Fussenegger

Technobiology's Enabler: The Magnetoelectric Nanoparticle Sakhrat Khizroev
Bioelectronic Medicine: From Preclinical Studies on the Inflammatory Reflex to New Approaches in Disease Diagnosis and Treatment

Valentin A. Pavlov, Sangeeta S. Chavan and Kevin J. Tracey

Vagus Nerve Stimulation and the Cardiovascular System Michael J. Capilupi, Samantha M. Kerath and Lance B. Becker

Harnessing the Inflammatory Reflex for the Treatment of Inflammation-Mediated Diseases Yaakov A. Levine, Michael Faltys and David Chernoff

Recording and Decoding of Vagal Neural Signals Related to Changes in Physiological Parameters and Biomarkers of Disease Theodoros P. Zanos

Restoring Movement in Paralysis with a

Bioelectronic Neural Bypass Approach: Current

State and Future Directions Chad E. Bouton

Bioelectronic Medicine--Ethical Concerns Samuel Packer, Nicholas Mercado and Anita Haridat

Use of Bioelectronics in the Gastrointestinal Tract Larry Miller, Aydin Farajidavar and Anil Vegesna

Vagus Nerve Stimulation at the Interface of BrainGut Interactions

Bruno Bonaz, Valérie Sinniger and Sonia Pellissier

For additional articles in this collection, see http://perspectivesinmedicine.cshlp.org/cgi/collection/ 